\title{
S-allyl dithiocarbazate: Cell viability and role of ligand on coordination
}

\author{
Zahra Yeke Ghasemi $^{1}$, Roberto Centore ${ }^{2}$, reza takjoo ${ }^{1}$ \\ ${ }^{1}$ Ferdowsi University Of Mashhad, Mashhad, Iran, Islamic Rep., ${ }^{2}$ University of Naples "Federico II", Via Cinthia, Naples, Italy \\ E-mail: yeke_z@yahoo.com
}

The coordination chemistry of dithiocarbazates as a class of Schiff bases has raised considerable interest because of their ability to form various metal complexes with different coordination numbers and geometries. These compounds have a wide range of properties including anticancer, antiprotozoa, DNA binding and cleavage, antibacterial, antifungal, antimalarial and insecticidal. Five dithiocarbazate complexes, CdL2 (1), ZnL2 (2), Cu2L2(OAc)2 (3), Cu2L2(NO3)2 (4) and CuLCl2 (5) have been synthesized using S-allyl-3-[(2-pyridyl-methylene)]dithiocarbazate $(\mathrm{HL})$ as tridentate ligand. HL crystallize in the space group P212121 with planar geometry and static disorder in the terminal allyl chain. It is interesting to compare the structural variations of the ligand, upon complexation in the structures 1-5 in which it is present in the deprotonated anionic form $\mathrm{L}^{\wedge}$-. In all the complexes, the ligand $L \wedge$ - coordinates to the metal through the pyridine nitrogen, the azomethine nitrogen and the thiolate sulfur atoms. Compounds 1 and 2 crystallize in the triclinic system with $\mathrm{P} 1^{-}$space group and the monoclinic system with $\mathrm{P} 21 / \mathrm{n}$ space group respectively. The compounds 1 and 2, two ligand units encompass the cadmium(II) and zinc(II) ions in bischelate distorted octahedral fashion. In the copper binuclear compound (3), each metal atom is coordinated to one $\mathrm{L}^{\wedge}$ - ligand unit and the two copper atoms are held by two bridging acetate ligands. The coordination number of copper is five, with distorted square pyramidal geometry. The copper in binuclear compound 4 show distorted octahedral geometry in which $L \wedge$ - and one $\mathrm{O}$ atom of nitrate ligand holds coordination axial positions and the equatorial sites are occupied with another $\mathrm{O}$ atom of nitrate ligand and thiolate sulfur atom of adjacent ligand. In this compound, the nitrate ligand act as bidentate and thiolate sulfur atom is bridge between two copper atoms. In the coordination polymer 5 , copper has a distorted square pyramidal geometry in which $L \wedge$ - holds three coordination positions and two chloride ligands complete the five-coordination arrangement, so that each chlorine atom links two adjacent $\mathrm{Cu}$ centers. The compound crystallizes in the acentric space group Pc. The cytotoxic activity of the three complexes (CdL2, CuL2(OAC)2 and CuLCl2) and the free ligand has been investigated by MTT assay, using cis-platin as comparative standard against several different human and animal cancer cell lines. The results evidence that cadmium and copper complexes induce apoptosis in cancer cell lines. Complex 1 exhibits the highest selectivity against the human breast cancer cells (MCF-7) and 5 shows the highest selectivity against bladder cancer cells (T-24). Complex 5 shows a high population of apoptotic cells (65.8\%), about two times higher than cis-platin (32.1\%) at the same concentration and can induce apoptosis in T-24 cancer cells. Likewise, complex 1 shows a high population of apoptotic cells $(55.2 \%), 1.2$ times higher than cis-platin (45.15\%) at the same concentration and can induce apoptosis in MCF-7 cancer cells.

[1] Takjoo. R. et al. (2012) J. Coord. Chem. 65, 1569-1579.

[2] Takjoo. R. et al. (2016) Inorg. Chim. Acta. 447, 52-58.

[3] Yazdanbakhsh. M. et al. (2009) J. Coord. Chem. 62, 3651-3660.

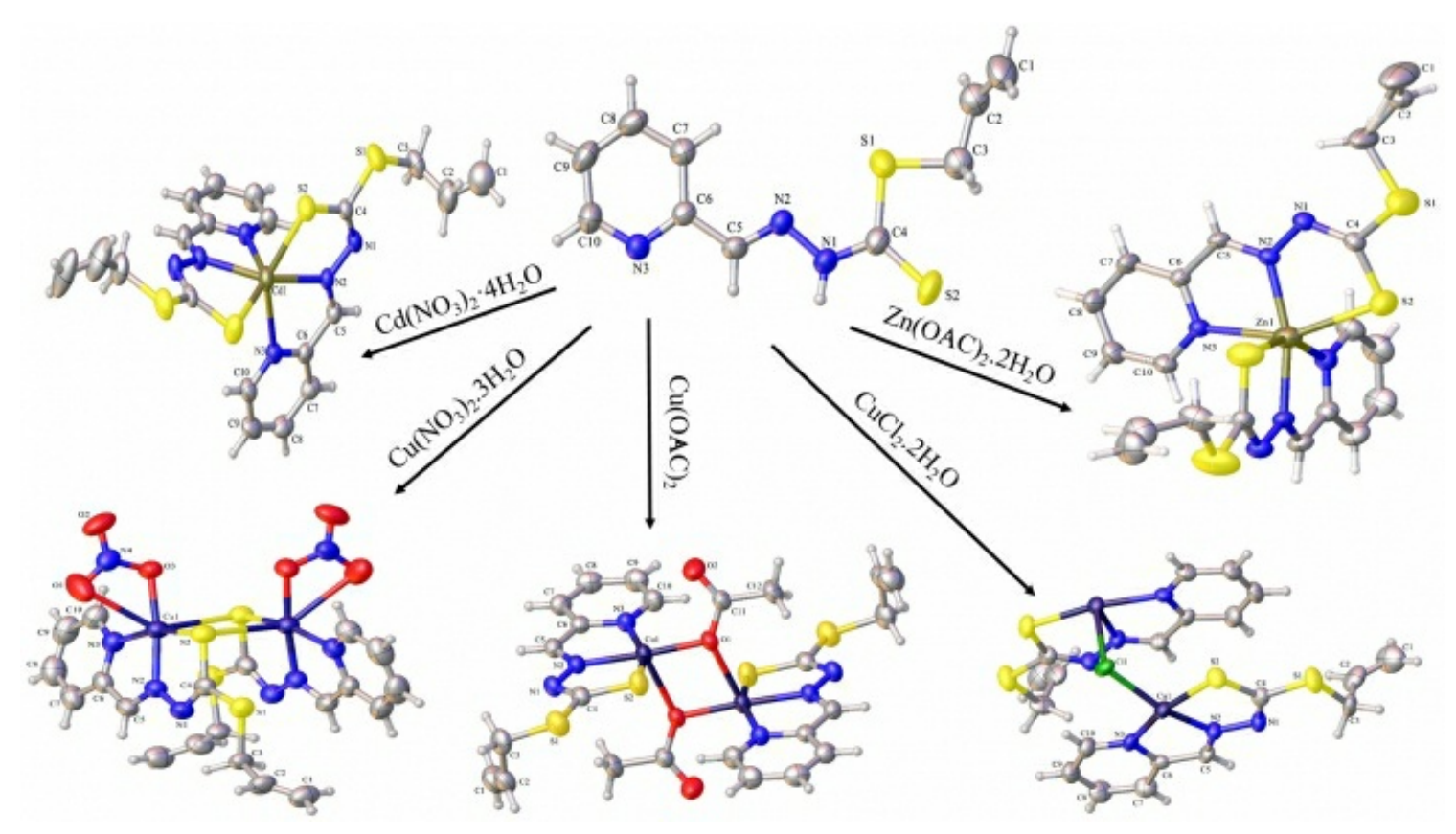

Keywords: Dithiocarbazate; Crystal structure; Cell viability 